\title{
On Entropy Measures for Dynamic Network Topologies: Limits to MANET
}

\author{
Roy Timo, Kim Blackmore and Leif Hanlen
}

\begin{abstract}
What are the fundamental limits on the communications potential of wireless networks? We contend that quantifying topological dynamics resulting from node movement enables one to: find the minimum overhead required by the network to maintain connectivity and, calculate the communication potential of the network.

A mobility metric is proposed for the unbiased comparison of networks. This is an entropy measure based on the uncertainty of change in the topology of the network, and is referred to as topological uncertainty. Topological uncertainty determines the minimum overhead required by the network to correctly identify the topology and hence, provide node connectivity. We use topological uncertainty to derive fundamental bounds on the maximum bit rate available within a Mobile Ad-Hoc Networking environment.

Our work demonstrates the potential of entropy measures to describe the complexities of node connectivity within wireless networks.
\end{abstract}

Index Terms- Entropy, Mobile Ad-Hoc Networks (MANET), Routing, Mobility Metrics, Throughput Capacity and Wireless Communications.

\section{INTRODUCTION}

Mobile Ad-Hoc Networking has evolved as a paradigm for the creation of robust "self-describing", "self-healing" networks [1-3]. Much mobile Ad-Hoc Networking literature [37] has focussed on the development of techniques for routing data through resource-constrained wireless environments. There has been little attention focussed on the causation of unavoidable routing overhead in dynamic topologies [8]. Inparticular, knowledge of the fundamental limits to communication potential are necessary to determine what can be achieved, what is a reasonable achievement, and how an optimal design solution should be approached.

MANET research today is typically conducted using empirical studies, for example [9], to estimate the abilities of proposed network connectivity schemes ${ }^{1}$. The performance of

Kim Blackmore is with the Department of Engineering, Faculty of Engineering and Information Technology, The Australian National University, Canberra ACT 0200, Australia. e-mail:Kim.Blackmore@anu.edu .au

Roy Timo is an undergraduate student with the Department of Engineering, Faculty of Engineering and Information Technology, Australian National University, Canberra ACT 0200, Australia. Part of this work was completed while visiting NICTA Canberra Laboratory. The remainder was completed as part of an honours undergraduate thesis for the Australian National University. email:u3310099@anu.edu.au .

Leif Hanlen is with National ICT Australia, Locked Bag 8001, Canberra ACT 2601 Australia, and affiliated with The Australian National University, Canberra ACT 0200, Australia. National ICT Australia is funded through the Australian Government's Backing Australia's Ability initiative, in part through the Australian Research Council. e-mail: Leif.Hanlen@nicta.com.au.

${ }^{1}$ Network connectivity schemes refer to networking infrastructure, in particular network layers 2 and 3. these schemes is evaluated via the use of the following parameters [10]: packet delivery ratio, end-to-end delay, path optimality, and throughput.

Designing connectivity schemes to optimize one or more of these parameters has shown to be a difficult task owing to the complex relationship between node movement, topological change, traffic patterns, multiple access and optimal route selection [11]. To reduce complexity, most researchers have adopted the Open System Interconnection (OSI) framework for network design $[11,12]$.

Conceived in the early 1970's, the OSI framework allows developers to divide network construction into isolated layers. Provided inter-layer dependency remains small ${ }^{2}$, this approach is very effective in isolating specific problems for rapid resolution [12]. However, when applied to more hostile networking environments, where inter-layer dependencies are much higher and capacity bounds much lower, this design principle has shown to be inadequate [4].

Motivated by this, MANET research is focussed on developing protocols specifically for the mobile wireless environment $[3,4]$. The theme of this research is retaining the OSI framework and developing MANET protocols within the existing structure. In particular, network layer routing protocols have received much attention. However, all MANET designs have exhibited increased overhead requirements associated with highly dynamic topologies [13]. When combined with the much tighter constraints of the wireless channel, this overhead introduces a situation where certain movement patterns may render a MANET infeasible. In other cases, the MANET may only be feasible for particularly efficient network designs.

In the present work, we argue that the sole unavoidable consequence of node mobility is a minimum overhead requirement necessary for the identification of the network's topology. Specifically, we contend that uncertainty in how the topology changes over time is a natural way to compare mobile networks and to quantify the identification process. Improved knowledge, or predictability of future topological change enables data to be routed more efficiently, potentially improving quality of service (QoS), and increases the spectral efficiency of the network.

The improvements to QoS and spectral efficiency available from the predictability of topological change have been demonstrated in recent work by $\mathrm{Su}$ et al. in $[14,15]$. In [14] an estimate of route failure time was derived from the likely future trajectory of each node calculated from their recent movements.

\footnotetext{
${ }^{2} \mathrm{~A}$ trait exhibited by many applications on static networks.
} 
This estimate was then used to optimally predict the time at which each route should be re-calculated. Such knowledge reduced the number of protocol control packets, and improved seamless route transitions across the dynamic topology required for realtime applications ${ }^{3}$. Additionally, [15] used mobility prediction to calculate the optimum refresh rate of a multicast routing protocol and hence minimize control packets. The routing protocols implemented in $[14,15]$ are "statistically" aware; that is to say, they exploit the statistical properties of the network to reduce the uncertainty of link changes, and hence improve performance.

From $[14,15]$ it is clear that many aspects of node movement are irrelevant when defining the complexity of networking within a mobile environment - if all nodes move very fast, but at the same speed and in the same direction, the topology does not change and static networking techniques would suffice. Hence, a measure of network mobility ${ }^{4}$, should be based on some aspect of topological change.

Currently, some MANET performance limitations result from imposing artificial structures - for example OSI - and are not indicative of fundamental communications potential of the mobile wireless environment. However, uncertainty in topological change promotes the need for network overhead that is fundamental to the MANET environment.

To improve network performance, we need to understand the limiting communications potential of MANETs.

- What is the maximum data a network can send?

- What is the minimum overhead required to describe the network over time?

The difference between these two values offers insight into the scalability and throughput characteristics of MANETs.

This paper is organized as follows. Section II explains the MANET operation and design environment. In section III we present a model that quantifies network change based on the work of Crutchfield et al. in [16]. This model is applied to a MANET operating over a Random Walk Mobility Model (RWMM) in section IV, which demonstrates that a Markov Process is an appropriate stochastic model for viewing topological change. Section V applies results developed in section III to evaluate the maximum available throughput for a RWMM MANET. Finally, section VI concludes and discusses future work.

\section{The MAnEt Environment, Simulation \& The RANDOM WALK MOBILITY MODEL}

\section{A. Connectivity in MANETs}

MANETs are decentralized, self-organizing, rapidly deployable, wireless networks, providing connectivity for a wide array of electronic devices. In their purest form, MANETs consist of a collection of mobile nodes which are distributed over a geographical area, who communicate discrete blocks of data, called packets, via radio links according to a set of predefined protocols. The uniqueness of MANETs is found in that each node has packet-switching capabilities and may simultaneously

\footnotetext{
${ }^{3}$ Real time applications have tight requirements on the maximum packet endto-end delay.

${ }^{4}$ A mobility metric.
}

represent a transmitter, receiver and a relay station [11]. Each node may use these capabilities to actively participate in the relaying of packets to destinations beyond the transmission range of the sender. As a result, packets may "relay" through several intermediate nodes along source-destination (S-D) routes. Additionally, as nodes are mobile, the stability of the wireless links interconnecting nodes can fluctuate substantially. Consequently, the topology of the network is dynamic, and to some degree unpredictable.

The connectivity service of the network needs to react to the random dynamic topology by identifying important topological change and accordingly adjust S-D routes. Under high traffic conditions it becomes increasingly important that route selection is optimized to efficiently use the available bandwidth. That is, route choices will inevitably need to exploit all facets of the changing node connectivity. To achieve this, the connectivity service requires accurate knowledge of the changing topology. If node movement contains some uncertainty, then topological change will necessarily exhibit uncertainty. The quantity or degree of uncertainty in topological change, topological uncertainty, refers to the extent to which connectivity patterns can be predicted.

If there is no uncertainty in node movement, the connectivity service can determine how the node connectivity will change, and therefore may devise optimal space-time [17] routes for each communication task. If however the connectivity service does not know with certainty what the future topologies will be, there is a need for additional data to be sent across the network. This additional data constitutes network overhead.

\section{B. Simulation}

As MANETs are yet to be realized, the vast majority of their development has been conducted within the simulation environment. Within this environment, proposed protocols are tested in hypothetical networks, with various signal attenuation schemes and traffic patterns. Node movement within these networks is typically defined according to a synthetic mobility model [18].

The primary distinction between MANETs and fixed wireless networks are the topological changes resulting from node movement. During simulation, it is typical to define a distance $R$, the node's transmission range, to represent the maximum distance at which an acceptable BER is achieved to allow for the signal to be understood. In this circumstance, topological change is a direct result of node movement. Characterizing topological change is therefore equivalent to characterizing node mobility.

\section{Mobility Metric}

A current open area of research belongs to the problem of defining a mobility metric to characterize the complexity of routing in the presence of node mobility. If defined, this metric would allow for the unbiased comparison of connectivity services across mobility models, and present an opportunity to design protocols to adapt to mobility changes. Proposed solutions include $[1,20,21]$. Such measures however, do not readily quantify the consequences of mobility in a manner that is reflected in the "best" performance available to any connectivity 
service. The best performance relates to the minimum transmitted information required by the connectivity service to describe the network, and naturally stems from the minimum information required to describe topological change. Thus, the entropy of topological change is a natural quantification of the effect of node mobility on network's connectivity service.

\section{Mobility Model}

The Random Walk Mobility Model (RWMM) is one of the simplest and most commonly used of the synthetic mobility models. In the RWMM, time is divided into a series of equal duration epochs [19], so that movement is discrete in time. At the start of an epoch each node moves from its current location to a new location, in a randomly selected direction $\phi$, at a randomly selected speed $v$.

After each movement phase, a pause time of duration $p$ occurs. To simplify calculation of the network throughput capacity, we only allow nodes to transmit during the pause time. All nodes are assumed to be identical, with identical communications abilities.

The use of the RWMM here is not intended to provide a realistic representation of mobility patterns expected in real-world applications. Rather it provides an opportunity to explore the limits on network performance under the most challenging situations where there is no discernable structure in the mobility patterns of nodes.

\section{DynAmic Networks, TOPOLOGICAL MOBILITy \& TOPOLOGICAL UNCERTAINTY}

Let $\Gamma_{i, j}^{t}$ be an indicator function representing the adjacency of node $j$ to node $i$ at time $t$.

$$
\Gamma_{i, j}^{t}= \begin{cases}1 & \text { link } i \rightarrow j \text { exists: } D(i, j) \leq R \\ 0 & \text { otherwise }\end{cases}
$$

where $D(i, j)$ indicates the distance between nodes $i$ and $j$.

A network of $N$ nodes can be described by the set of indicator variables $\Gamma_{i, j}^{t}$ for $i, j \in 1,2, \ldots, N$. We note that a node cannot be adjacent to itself ${ }^{5}$ and accordingly define $\Gamma_{i, j}^{t} \equiv 0$ for all $i=j$. We let the set of indicator variables $\Gamma_{i, j}^{t}$ form an $N \times N$ adjacency matrix $A^{t}$, where $A_{i, j}^{t} \equiv \Gamma_{i, j}^{t}$. The adjacency matrix $A^{t}$ represents the entire topology of the network at time $t$. Each node has identical transmission power, and hence transmission range, so each link is bi-directional with $\Gamma_{i, j}^{t}=\Gamma_{j, i}^{t}$. Thus $A^{t}$ is a binary symmetric matrix.

The set of allowable configurations of $A^{t}$ forms a state space $S \equiv s_{1}, s_{2}, s_{3}, \ldots, s_{\gamma}$, where

$$
\gamma=2^{\frac{N(N-1)}{2}}
$$

At any one time the network must assume exactly one of these states; that is, $A^{t}=s_{k}$ for some $0 \leq k \leq N$.

Let $X$ be a random process denoting the trajectory of the networks topology through the state space $S$, and let $x$ denote a particular instance of $X$. The changes occur at discrete points in time, so $X$ is a discrete random process. A causal network connectivity service may gain an understanding of topological change by considering the past history of topological change.

\footnotetext{
${ }^{5}$ cannot transmit a message to itself
}

\section{A. Fundamental Randomness}

Fundamental randomness of topological change quantifies the non-deterministic aspect of a network's topology, and provides an estimate of the likelihood of a particular topology occurring at a future time, given the topological history.

Let

$$
\zeta^{t}:=\left\{\zeta_{0}^{t}, \zeta_{1}^{t}, \zeta_{2}^{t}, \ldots, \zeta_{\gamma-1}^{t}\right\}
$$

represent the probability distribution across the state space $S$, at time $t$ where $\zeta_{i}^{t} \equiv P\left(x^{t}=s_{i}\right)$. The probability of a particular state occurring at the $(t+1)^{t h}$ epoch is conditionally dependent on the entire past:

$$
\zeta_{i}^{t+1}=P\left(x^{t+1}=s_{i} \mid x^{-\infty}, \ldots, x^{t}\right) P\left(x^{-\infty}, \ldots, x^{t}\right)
$$

Equation 4 implies, if there is some relationship between the past and the future, then there is potential in using knowledge of the past to improve prediction of the future. If we assume that the network connectivity service is limited to considering only a finite number of states $k$, in its prediction of future states, we may view topological change as a $k$-step Markov Process. In section IV, we demonstrate that the majority of this past-future dependance under the RWMM is described by a 1-step Markov process. Furthermore, for $k \geq 2$ we find diminishing reductions in uncertainty and thus contend there is limited benefit in pursuing increasing complex models for state change.

Given the state space $S$, and probability distribution $\zeta$, the average information gained when a state is revealed to the connectivity service is given by Shannon's entropy[22]:

$$
\begin{aligned}
H(x) & =-\sum_{s_{i} \in S} P\left(s_{i}\right) \log _{2} P\left(s_{i}\right) \\
& =-\sum_{i=0}^{\gamma-1} \zeta_{i} \log _{2} \zeta_{i}
\end{aligned}
$$

\section{B. Notation}

Definition 1-L-Block Sequence: Let

$$
\vec{x}^{L}=x^{t}, x^{t+1}, \ldots, x^{t+L-1}
$$

denote a block of $L$ consecutive states which the network visits starting after the $t^{t h}$ epoch, where each $x^{t}$ is selected from the networks state space $S$ with probability distribution $\zeta^{t}$.

We shall shortly consider the limit of an L-Block sequence, as $L$ becomes countably infinite.

Definition 2-Probability of L-Block Sequence: Let $P\left(\vec{x}^{L}\right)=P\left(x^{t}, x^{t+1}, \ldots, x^{t+L-1}\right)$ denote the joint probability of $L$ consecutive states starting after the $t^{t h}$ epoch.

Definition 3-Uncertainty of L-Block Sequence: The uncertainty of the sequence of $L$ states is given by Shannon's block entropy:

$$
H\left(\vec{x}^{L}\right) \equiv-\sum_{x^{t} \in S} \ldots \sum_{x^{t+L-1} \in S} P\left(\vec{x}^{L}\right) \log _{2} P\left(\vec{x}^{L}\right)
$$

The entropy of all possible sequences of topologies, for the network $X$ over all time, is determined by evaluating $H\left(\vec{x}^{L}\right)$ for $L \rightarrow \infty$. 
We assume that there is some non-zero message traffic across the entire topology of the network during the pause time after each epoch; that is to say, each node in the network engages in one or more of the following tasks: transmission, forwarding or receiving of messages. It is therefore necessary for the network to completely resolve the topological uncertainty introduced from the last epoch, prior to communications taking place. This incremental increase in entropy from epoch to epoch, quantifies the non-deterministic aspects of topological change resulting from node movement. We view the entropy rate of the network as a fundamental randomness measure, or mobility noise floor that is a quantifiable characteristic of the network's mobility model. For example, we will see in Figure 1 that as node speed in the RWMM is increased, the mobility noise floor or entropy rate of the network also has a corresponding increase.

The entropy rate $h_{\mu}$ of the network is:

$$
h_{\mu}=\lim _{L \rightarrow \infty} \frac{H\left(\vec{x}^{L}\right)}{L}
$$

It is not possible for the network connectivity service to improve topological state predictability beyond $h_{\mu}$ by analyzing topological data alone. Thus we may say, $h_{\mu}$ represents an optimal level of state predictability.

\section{Topological Uncertainty}

An optimally predictive connectivity service requires complete past state information: either by direct measurement, or $a$ priori knowledge of a stochastic process model for the topological change. Sub-optimal arrangements are possible by neglecting model aspects.

For example, a connectivity service that ignores all statistical properties of state sequences, assumes that each of the $\gamma$ states from Equation 2 are equally likely, and thus faces the maximum uncertainty $\log _{2}(\gamma)$ when topological change occurs with each epoch. On the other hand, a connectivity service that has $a$ priori knowledge of the stochastic process governing topological change will experience the minimum uncertainty $h_{\mu}$ from epoch to epoch. We are interested in how this uncertainty converges from $\log _{2}(\gamma)$ to $h_{\mu}$ as the stochastic model used by the connectivity service improves. Crutchfield dealt with such issues in a unifying paper on entropy convergence [16]. In what follows, we analyze topological change using the same mathematical framework developed in [16], and for the sake of clarity and uniformity, adopt the same notation.

Practical connectivity services cannot derive infinite block length statistics, they must employ a finite-L approximation to $h_{\mu}$, such as the topological uncertainty $\widehat{h_{\mu}}$ :

$$
\widehat{h_{\mu}} \equiv H\left(\vec{x}^{L}\right)-H\left(\vec{x}^{L-1}\right) \text {. }
$$

Note that the topological uncertainty converges faster to $h_{\mu}$ than $\frac{H\left(\vec{x}^{L}\right)}{L}$ does [16]. It is for this reason we adopt Equation 8 as our definition of topological uncertainty.

\section{Predictability Gain}

The predictability gain in going from length $L$ statistics, to length $L+1$ statistics is:

$$
\Delta \widehat{h_{\mu}}(L) \equiv \widehat{h_{\mu}}(L)-\widehat{h_{\mu}}(L-1)
$$

where the total predictability $G$, of the dynamic topology is:

$$
G \equiv \sum_{i=1}^{\infty} \Delta \widehat{h_{\mu}}(L)=-\left(\log _{2}(\gamma)-h_{\mu}\right)
$$

Predictability gain quantifies (in bits) the predicability improvement in going from length $L$ statistics to length $L+1$ statistics. It also offers a way to quantify the spectral efficiency benefit made possible by improving the statistical model of the network against the increased computational load of the more complex model. In the following section we estimate the predictability of a network by counting the relative frequency of $L$ states over a large number of epochs ${ }^{6}$.

\section{Markovian Nature of Topological Change UNDER THE RWMM}

This simulation demonstrates that RWMM defines a Markovian dynamic topology process with transition matrix $T$ and steady state distribution $\pi$. The topology of a network connecting 3 nodes moving according to the RWMM was calculated after each epoch for $10^{5}$ epochs. This gave a trajectory of $10^{5}$ states. All 3 nodes were assumed to have the same transmission range $R$, and velocity $v$. The terrain for the network as a square of unit area, whose edges were folded over to form a torus. This avoided the introduction of additional "boundary conditions", such as bouncing off terrain boundaries to the RWMM profile.

Figure 1 illustrates the rapid decrease of $\widehat{h_{\mu}}$ for four node speeds, $v=0.1,0.05,0.01,0.005$ as the complexity of the statistical model is increased (increasing block lengths $L$ ). A 1step Markov Process has the property that $\widehat{h_{\mu}}$ converges to $h_{\mu}$ at a block length 2 [16]. We observe that beyond a block length of $2 \widehat{h_{\mu}}$ continues to decrease slowly. We conjecture that $\widehat{h_{\mu}}$ will not converge to an asymptotic value $h_{\mu}$ at block length 2 .

The rapid decrease of $\widehat{h_{\mu}}$ up to block length 2 and slow decrease thereafter strongly suggest a 1-step Markov Model is a simple and accurate stochastic model for topological change for a RWMM. Thus we set $h_{\mu}$ equal to the entropy rate of the corresponding 1-step Markov Process and absorb any further dependencies as apparent randomness. This simplification allows us to estimate the entropy rate $h_{\mu}$ (mobility noise floor) of the network by considering state transitions only; that is, block lengths $L=2$. Intuitively, we can think of this outcome as resulting from the movement of each node being independent from epoch to epoch and therefore network connectivity being largely independent beyond that of the connectivity of the epoch. As node speed is increased, we see a corresponding increase in the network mobility noise floor $\left(h_{\mu}\right)$, and thus an increase in the minimum overhead required by the network. This result is expected to be characteristic of mobility models where there is no movement correlation between nodes.

\section{Fundamental Limits on Communication}

The topological uncertainty $\widehat{h_{\mu}}$ is the uncertainty experienced by a real connectivity service employing finite length

\footnotetext{
${ }^{6} \mathrm{We}$ assume that there are no cyclic structures in topological change with period greater than that of the largest block length tested.
} 
Fig. 1. Convergence of topological uncertainty $\widehat{h_{\mu}}$ to entropy rate $h_{\mu}$.

statistics. In this section we consdier the fundamental bounds $\widehat{h_{\mu}}$ places on the total network throughput for the RWMM. The topological uncertainty is the information that must be measured by the connectivity service via some measurement channel after each epoch to correctly identify the topology of the network. So at least $\widehat{h_{\mu}}$ bits must be transmitted after each epoch to account for the randomness introduced by node movement.

\section{A. Throughput Capacity}

Let $c$ bits sec denote the maximum transmission rate possible over the wireless channel from any node $i$ to any adjacent node $j$. That is to say, each node can either transmit or receive $c$ bits sec to or from any adjacent node respectively. Thus, the uppermost limit on throughput available to the network is given by the total number of transmitting-receiving node pairs; that is:

$$
C \leq c \frac{N}{2} \quad \frac{\text { bits }}{\text { sec }}
$$

where $N$ is the number of nodes in the network.

\section{B. Available Throughput}

If $N$ is increased, the amount of information a routing protocol needs to describe the network can be expected to increase. If communication is allowed only during the pause time $p$, the minimum overhead $O_{\min }$ in bits sec is:

$$
O_{\min }=\frac{\widehat{h_{\mu}}}{p} \quad \frac{\text { bits }}{s e c}
$$

The average available space for useful (higher layer) data is found by subtracting the minimum network overhead $O_{\min }$ from the network throughput capacity $C$.

$$
C_{\text {avail }}=C-O_{\min } \quad \frac{\text { bits }}{\text { sec }}
$$

For example, consider a network connectivity service that does not consider statistical data when identifying topological change. Such a service will only be optimal if all $\gamma$ states are in fact equally likely. The service implicitly assumes that all $\gamma$ states are equally likely, and thus the topological uncertainty experienced by the service is always a maximum (no matter what the underlying distribution of states actually is):

$$
\begin{aligned}
\widehat{h_{\mu}} & =\log _{2}(\gamma) \\
& =\frac{N(N-1)}{2}
\end{aligned}
$$

From Equations 13 and 14 the average network available network throughput capacity for such a service no more than

$$
C_{\text {avail }}=c \frac{N}{2}-\frac{N(N-1)}{2 p}
$$

Equation 15 implies that the maximum number of nodes that can be supported by a MANET without statistical topology awareness is

$$
N_{\max }=c p+1 \text {. }
$$

This result is analogous to the well known maximal entropy for equi-probable events in discrete entropy.

\section{Scaling of a RWMM MANET}

A MANET with zero topological uncertainty has a minimum network overhead of 0 bits sec for all $N$. On the other hand, a MANET with maximum topological uncertainty, has a minimum network overhead requirement that grows with respect to $N$ at a rate greater than the maximum network throughput capacity.

We demonstrate that, for a MANET whose nodes move according to a RWMM, the minimum overhead imposes an upper bound on the uncertainty in state transitions.

From Section IV, we know that the topology of the network is a Markov Process. Therefore the topological uncertainty is

$$
\widehat{h_{\mu}}=\sum_{i=0}^{\gamma-1} \sum_{j=0}^{\gamma-1} \pi_{i} T_{i, j} \log _{2} \frac{1}{T_{i, j}}
$$

where $\pi$ is the steady state vector and $T$ is the transition matrix respectively associated with the Markov Process. Let the smallest uncertainty for each of the possible state transitions be

$$
\epsilon_{\min }=\min _{0 \leq i, j \leq \gamma-1}\left\{\pi_{i} T_{i, j} \log _{2} \frac{1}{T_{i, j}}\right\} \quad 0 \leq i, j \leq \gamma-1 .
$$

Then the topological uncertainty is bounded by

$$
\widehat{h_{\mu}} \geq \gamma^{2} \epsilon_{\min }
$$

Comparing with 12 , this gives a lower bound on the minimum overhead.

From equation 13, we obtain an upper bound on the available throughput for the network

$$
C_{\text {avail }} \leq c \frac{N}{2}-\frac{1}{p} \gamma^{2} \epsilon_{\min } \quad \frac{\text { bits }}{s e c}
$$


This upper bound is negative when

$$
\epsilon_{\min }>\frac{c p N}{2 \gamma^{2}}
$$

Using equation 2, equation 18 becomes:

$$
\epsilon_{\min }>\frac{c p N}{(2) 2^{N^{2}-N}}
$$

That is, the available throughput is zero if all of the state transitions exhibit uncertainty greater than $\frac{c p N}{(2) 2^{N^{2}-N}}$. This limit tends to 0 as $N \rightarrow \infty$.

So for any $\epsilon_{0}$, there exists some number $N_{0}$ such that a network with more than $N_{0}$ nodes and minimum state transition uncertainty $\epsilon_{0}$, has available throughput zero. This indicates that the RWMM MANET cannot support infinitely large networks.

\section{CONCLUSION}

A mobility metric based on the topological uncertainty of the network was proposed. This metric is an application of the finite L-block approximation to the entropy rate of the topology considering topological change as a random process. This measure provided the minimum information required to describe topological change from epoch to epoch and thus constituted a minimum overhead to quantify node mobility. The mobility metric was used to describe scaling properties of MANETs under the random walk mobility model. We demonstrated that topological change resulting from the random walk mobility model is accurately described by a Markov process. These results suggest potential benefits in exploring statistically aware network designs. Future work will develop models for non stationary (statistically) topologies and explore the use of predictability gain measures as justification for increasing computational load.

\section{REFERENCES}

[1] Bruce McDonald, "Link availability models for mobile ad-hoc networks,"technical report TR99-07, University of Pittsburgh, Department of Computer Science, May 1999.

[2] A. Alwan, R.Bagrodia, N. Bambos, M.Gerla, L. Kleinrock, J. Short, and J. Villasenor. "Adaptive Mobile Multimedia Networks", IEEE Personal Communications Magazine, vol. 3, no. 2, pp. 34-51, April 1996.

[3] C. Perkins, Ad Hoc Networking, 1st ed. Addison-Wesley, Dec. 2000.

[4] E. Royer and C. Toh, "A Review of Current Routing Protocols for Ad-Hoc Mobile Wireless Networks," IEEE Personal Communications Magazine, pp. 46-55, April 1999.

[5] P. Gupta and P. Kumar, "A System and Traffic Dependent Adaptive Routing Algorithm for Ad Hoc Networks," proc. IEEE 36th conf. on Decision and Control, pp. 2375-2380, San Diego, 1997.

[6] G. Aggelou and R. Tafazolli, "A bandwidth-efficient Routing Protocol for Mobile Ad hoc Networks," proc. ACM International Workshop on Wireless Mobile Multimedia (WoWMoM), pp. 26-33, Seattle, August 1999.

[7] A. Kashyap, H. Nishar, and P. Agarwal, "Survey on unicast routing in mobile ad hoc networks," http://citeseer.ist.psu.edu/481660.html

[8] R. Timo. "Fundamental Limits on Performance of Mobile Ad-Hoc Networks Derived from Topological Uncertainty." Honour's thesis, Australian National University, 2004.

[9] J. Broch, D. Maltz, D. Johnson, Y. Hu, J. Jetcheva, "A Performance Comparison of Multi-HopWireless Ad Hoc Network Routing Protocols," proc. 4th Annual ACM/IEEE International Conference on Mobile Computing and Networking, pp.85-97, Dallas, October 1998.

[10] M. Subbarao, "Ad Hoc Networking Critical Features and Performance Metrics," technical report Wireless Communications Group NIST, October 1997.
[11] M. Subbarao, "On Optimizing Performance in Mobile Packet Radio Networks." PhD Thesis, The John Hopkins University, 1998.

[12] A. Ephremides and B. Hajek, "Information Theory and Communication Networks: An Unconsummated Union," IEEE Transactions on Information Theory, vol. 44, no. 6, pp. 2416-2434, 1998.

[13] L. Viennot, P. Jacquet and T. Clausen, "Analyzing control traffic overhead versus mobility and data traffic activity in mobile Ad-Hoc network protocols," Wireless Networks, vol. 10, no. 4, pp. 447-455, July 2004

[14] S. Lee, W. Su and M. Gerla, "Mobility Prediction and Routing in Ad Hoc Wireless Networks," technical report Wireless Adaptive Mobility Laboratory, Deptartment of Computer Science, University of California, Los Angeles, $C A$.

[15] S. Lee, W. Su and M Gerla, "Wireless Ad-Hoc Multicast Routing with Mobility Prediction," Mobile Networking Applications, 6(4), pp. 351-360, 2001.

[16] J. Crutchfield and D. Feldman, "Regularities Unseen, Randomness Observed: Levels of Entropy Convergence," Santa Fe Institute Working Paper 01-02-012, arXiv.org/abs/cond-mat/0102181, February 2001.

[17] P. Jacqueta and D Voluceau, "Space-Time Information Propagation in Mobile Ad-Hoc Wireless Networks," proc. 2004 IEEE INFORMATION THEORY WORKSHOP San Antonio, October, 2004.

[18] T. Camp and J. Boleng and V. Davies, "A Survey of Mobility Models for Ad Hoc Network Research," Wireless Communications \& Mobile Computing (WCMC): Special issue on Mobile Ad Hoc Networking: Research, Trends and Applications, vol. 2, no. 5, pp 483-502, 2002.

[19] S. Xu, K. Blackmore and H. Jones, "Movement and Link Analysis of Mobile Node in Ad Hoc Networks," technical report Deptartment of Engineering, Australian National University, Feburary 2004.

[20] J. Tsumochiy, K. Masayamayy, H. Ueharay and M. Yokoyama, "Impact of Mobility Metric on Routing Protocols for Mobile Ad Hoc Networks," proc. IEEE Pacific Rim Conference on Communications, Computers and Signal Processing (PACRIM03), pp. 322-325, August 2003.

[21] X. Perez-Costa, C. Bettstetter and H. Hartenstein, "Towards a mobility metric for comparable \& reproducible results in ad hoc networks research (poster abstract)," ACM Mobile Computing and Communications Review (MC2R), vol. 7, no. 4, October 2003.

[22] C. E. Shannon, "A Mathematical Theory of Communication", Bell System Tech. J., vol. 27, pp. 379-423 623-656, 1948. 